\title{
Putting health at the center of climate action
}

\author{
The Glasgow Climate Pact reaffirms countries' commitments to limiting global heating to $1.5^{\circ} \mathrm{C}$, but without \\ prioritizing health, equity and multisectoral engagement within climate action, the health of the planet and the \\ future of humanity remain at grave risk.
}

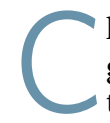

limate change is undoubtedly the greatest threat facing humanity in the twenty-first century. Highlighting the urgency and gravity of the issues at stake, the 26th annual Conference of the Parties of the United Nations Framework Convention on Climate Change (COP26) was held in Glasgow, UK, 31 October to 12 November 2021, in the midst of the ongoing COVID-19 pandemic. The meeting concluded with the agreement of the Glasgow Climate Pact, intended to accelerate the pace of global climate action and affirm commitments to limiting temperature rises to $1.5^{\circ} \mathrm{C}$. The pact acknowledges that climate change is a shared concern across humankind and urges parties to consider their obligations on human rights, the right to health and the rights of underserved and vulnerable populations when taking climate action. Although the pact recognizes the impacts of rising temperatures and extreme weather as social, economic and environmental threats, the lack of mention of the catastrophic effects on human health and wellbeing is a glaring omission.

The risks to health, and to fair and equitable societies, that are associated with climate change are multifaceted and well established. Air pollution, for example, is a major contributor to chronic respiratory conditions and cardiovascular disease and is known to disproportionately affect vulnerable populations, such as groups of low socioeconomic status, who are more likely to live in areas with poor air quality. Sustainable food systems are fundamental to raising populations out of poverty, in addition to supporting healthy diets and preventing the global rise of diabetes, obesity and malnutrition. Biodiversity loss and harmful livestock and agricultural practices contribute to greenhouse gas emissions and threaten the resilience of food systems, as well as driving zoonoses and disease outbreaks.

The World Health Organization (WHO) COP26 special report - The Heath Argument for Climate Action - launched at a dedicated session on health and climate at
COP26, underscores the direct and indirect effects of climate change on human health. Although the arguments presented are not new, the report calls for the integration of health as a central component of global climate action and alignment of the goals of the Paris Agreement with those of the WHO Manifesto, through leveraging of efforts in the global recovery from COVID-19. Indeed, as early as May 2020, health professionals worldwide recognized the opportunity for greater investments in health and environmental stewardship in pandemic recovery programs in an open letter to the G20 Summit. And in the lead up to the 2021 United Nations General Assembly, editors from over 200 health journals called for swifter action on climate change, highlighting the disproportionate burden of harm that global heating (largely driven by wealthy countries) inflicts on lowand middle-income countries and on the most vulnerable members of societies.

Although it has long been known that addressing climate change is mutually beneficial to improving human health and reducing inequities, the issue of how to implement policies that drive tangible action remains an open one. The ten recommendations within the WHO COP26 special report highlight opportunities for governments and other stakeholders to implement solutions while at the same time placing health equity and justice at the center of the response to the ongoing climate crisis. Encouragingly, the report goes beyond rhetoric and presents a plethora of case studies that showcase country-level and regional-specific practical initiatives to combat climate change and promote healthy outcomes.

One such example is the Urban Health Initiative pilot program in Accra, Ghana. A collaborative effort of the WHO, the Urban Health and Short-Lived Climate Pollutants Reduction Project and local government, this project aims to integrate and embed health policies into traditionally non-health governmental sectors, such as household energy, transport and solid-waste management. Cross-sector policy development in Accra has been achieved through a six-step process beginning with assessment of air pollution levels and sources in the city and identifying key stakeholders and sectors that have an impact on air quality. Focus then turned to building capacity across sectors to engage stakeholders and to communicate with the public and policymakers on the links between air pollution and health outcomes, as well as training staff to implement tools for assessing health and economic impacts of policies. Progress in both air pollution and health outcomes are being monitored with a policy-tracking framework. With the availability of tools and building capacity to tackle climate change and improve community health, Accra is now an exemplar city for the integration of health policies across multiple sectors of government.

The case studies presented in the WHO report each represent a nuanced implementation of policy designed to support and build capacity in a given region. But there are common actions that can be tailored for application in different settings. Training experts in communication to work across governmental departments, as well as fostering grassroots engagement, particularly with underserved populations, are fundamental to embedding health and equity into climate action. Although healthcare professionals, civil society and others have called for a health-centered approach, the United Nations and WHO are best positioned to work with Member States to support health-focused multisectoral policies, particularly in the global recovery from COVID-19. The health of the planet and of humanity are intrinsically entwined - a health-centered approach to climate action ensures that what is good for the planet is good for humanity.

Published online: 24 January 2022 https://doi.org/10.1038/s41591-021-01658-2 